\title{
Euglycemic diabetic ketoacidosis reported in pregnant women.
}

\author{
Balasubramaniam Branavan, Selladurai Pirasath, Athwel Gamarallage Hemal Sugathapala \\ Colombo South Teaching Hospital, Kalubowila, Sri Lanka.
}

Copyright: This is an open-access article distributed under the terms of the Creative Commons Attribution $\underline{\text { License, }}$, which permits unrestricted use, distribution, and reproduction in any medium, provided the original $\overline{\text { author and source are credited (CC BY 4.0) }}$

\section{Abstract}

Pregnancy is a physiological state in women which creates significant alterations in energy metabolism to provide energy supply to the foetus. The maternal fasting glucose level would be less than that of a non-pregnant state and in contrast plasma ketones and free fatty acids levels are elevated, resulting in a state of accelerated starvation. These metabolic alterations place a pregnant woman at a higher risk of developing euglycaemic ketoacidosis. We report a rare case of euglycaemic ketoacidosis causing severe increased anion gap metabolic acidosis in a pregnant woman.

Correspondence Email: hello.brana@live.com

Keywords: Euglycemic ketoacidosis, pregnancy

\section{Introduction}

Diabetic Ketoacidosis (DKA) is defined as presence of moderate serum or urine ketone bodies, serum glucose above $250 \mathrm{mg} / \mathrm{dL}$, arterial $\mathrm{pH}$ less than 7.3 and serum bicarbonate below $18 \mathrm{mEq} / \mathrm{L}$ (1) and it causes serious metabolic complication with high mortality rate. DKA can occurs in pregnancy and lead to compromise in both the mother and fetus (2). The hormonal changes in pregnancy create a state of insulin resistance allowing free flow of glucose to the foetus. Thus, prolonged starvation will also place her at high risk of starvation ketosis.

Here we present a pregnant patient with type 1 diabetes presenting with euglycemic diabetic ketoacidosis.

\section{Case report}

A 29year old primigravida mother was presented at her 29 weeks of gestation to surgical ward with fever, left sided lower limb swelling and persistent vomiting for 2 days duration. Initial obstetric evaluation was focused on excluding possibility of an obstetric emergency. She had type 1 diabetes mellitus which required bolus insulin regime three times daily and basal bolus regime at night. A clinical diagnosis of cellulitis was made and was treated with intravenous clindamycin. Upon examination, she was found to be unwell and persistently tachypneic; however, her haemodynamic status was stable. Her capillary blood sugar was $135 \mathrm{mg} / \mathrm{dL}$ and ABG showed $\mathrm{pH}$ of $7.1, \mathrm{pCO} 2$ of $7.2 \mathrm{mmHg}, \mathrm{p} 02$ of $110.5 \mathrm{mmHg}$, $\mathrm{Hco} 3$ - of $10.5 \mathrm{mmol} / \mathrm{L}$ and lactate of $0.9 \mathrm{mmol} / \mathrm{L}$. Urine ketone bodies were $3+$. Serum electrolytes were $\mathrm{Na}+$ $138 \mathrm{mmol} / \mathrm{L}, \mathrm{K}+-3.3 \mathrm{mmol} / \mathrm{L}$ and $\mathrm{Cl}--100 \mathrm{mmol} / \mathrm{L}$. She had persistently low lactate level with normal liver biochemistry and renal profile which made sepsis a remote possibility to cause severe increased anion gap metabolic acidosis. However, further metabolic workup related to acidosis showed euglycemic ketoacidosis and she was managed according to usual diabetes ketoacidosis protocol. Fluid resuscitation with $1 \mathrm{~L}$ of $0.9 \%$ saline was given over one hour and later converted to $0.45 \%$ saline and $5 \%$ dextrose infusion at a rate of $250 \mathrm{~mL} /$ hour for next 6hours and later converted to $100 \mathrm{~mL} /$ hour infusion. Intravenous soluble infusion $(0.1 \mathrm{U} / \mathrm{hr})$ was started at $6 \mathrm{U} / \mathrm{hr}$ with intravenous potassium replacement. We continued insulin infusion for nearly 72 hours until patient improved with no vomiting and improved appetite with negative urine ketone bodies. Later insulin was converted to subcutaneous soluble insulin.

\section{Discussion}

Patients with type 1 diabetes can present with features of diabetic ketoacidosis without hyperglycemia which is 
defined as euglycemic diabetic ketoacidosis. Reduced hepatic production of glucose during fasting or increased urinary excretion of glucose by counter regulatory hormones causes euglycemic diabetic ketoacidosis (3). Infections, poor adherence to treatment, persistent vomiting, corticosteroid therapy and beta sympathomimetic drugs are common precipitating factors for DKA in pregnancy (2). The patient with a trigger for diabetic ketoacidosis, taking regular insulin levels despite not taking meals, liver in a state on depleting glycogen causes less glucose production. Moreover, lipolysis with fatty acid production causes ketone production and lead to euglycemic diabetic ketoacidosis (3). Common causes of euglycemic DKA are pregnancy, low calorie intake, fasting or starvation, pancreatitis, SGLT2 inhibitors and prolonged vomiting (4). In our case, poor intake of meals due to infection, despite taking regular insulin causes lipolysis and subsequently more productions of ketone bodies lead to euglycemic diabetic ketoacidosis.

Diagnosis of euglycemic diabetic ketoacidosis is made after exclusion of other causes of high anion gap metabolic acidosis. In our case, patient's lactate level is normal. Patient did not have evidence of renal failure or any ingestions of alcohol or salicylates. Early clinical suspicion of euglycemic diabetic ketoacidosis and prompt treatment lead to less mortality. Treatment is same as management of diabetic ketoacidosis. Treatment compromise of adequate fluid resuscitation, continuing insulin with dextrose infusion until clinical improvement, normalizing bicarbonate and anion gap. (5)

\section{Declaration of conflicting}

\section{Interests}

The authors declared no potential conflicts of interest with respect to the research, authorship and/or publication of this article.

\section{Informed consent}

Informed written consent was obtained from the patient for her anonymized information to be published in this article retrospectively.

\section{References}

1. Nyenwe EA, Kitabchi AE. The evolution of diabetic ketoacidosis: an update of its etiology, pathogenesis and management. Metabolism 2016; 65: 507-21.

2. Kamalakannan D, Baskar V, Barton DM, et al. Diabetic ketoacidosis in pregnancy Postgraduate Medical Journal 2003;79:454-7.

3. Kitabchi AE, Umpierrez GE, Miles JM, Fisher JN. Hyperglycemic crises in adult patients with diabetes. Diabetes Care 2009;32: 1335-43.

4. Rawla P, Vellipuram A, Bandaru S, Pradeep Raj J. Euglycemic diabetic ketoacidosis: a diagnostic and therapeutic dilemma. Endocrinology, Diabetes \& Metabolism Case Reports 2017;15: 201-7.

5. Gelaye A, Haidar A, Kassab C, Kazmi S , Sinha P. Severe ketoacidosis associated with canagliflozin (Invokana): a safety concern. Case Reports in Critical Care 2016; 2016:1656182. 